\title{
Mastoid and Epitympanic Obliteration in Canal Wall Up Mastoidectomy for Prevention of Retraction Pocket
}

\author{
*Won Sang Lee, *Jae Young Choi, *Mee Hyun Song, *Eun Jin Son, \\ $\uparrow$ Sang Ho Jung, and *Sung Huhn Kim \\ *Department of Otorhinolaryngology, Yonsei University College of Medicine, Seoul, and †Department of \\ Otorhinolaryngology, Yonsei University Wonju College of Medicine, Wonju, Korea
}

Objective: To evaluate the surgical outcome in patients who have undergone mastoid and epitympanic obliteration technique.

Study Design: Retrospective review.

Setting: Tertiary care referral center.

Patients: The study group included 151 patients with cholesteatoma who underwent mastoid and epitympanic obliteration technique. This technique was applied to cases who had a strong chance of recurrent retraction pocket and cholesteatoma formation, including those patients with an adhesive drum indicating poor eustachian tube function $(\mathrm{n}=$ $52)$ and patients with a destructive scutum $(n=68)$. Thirty-one patients had both an adhesive drum and a destructive scutum. Intervention: The connection between the mastoid cavity and the middle ear was blocked by obliterating the epitympanum and antrum with bone pate and the remaining mastoid cavity with abdominal fat.

Main Outcome Measures: The postoperative drum state, the incidence of retraction pocket formation and cholesteatoma recurrence, the surgical complications of obliteration, and the hearing outcome.

Results: In 114 of 151 patients (75.4\%), the middle ear was well healed and well aerated. The retraction pocket formation or cholesteatoma recurrence did not develop in any subject. Postauricular skin depression was the most common complication of this technique $(\mathrm{n}=31$ [20.5\%]). In three patients $(2.0 \%)$, the bone pate used for obliteration was infected. Of the 56 cases who underwent a staged operation to regain their hearing, 37 resulted in a postoperative air-bone gap less than $20 \mathrm{~dB}$ hearing level.

Conclusion: Mastoid and epitympanic obliteration is an effective option for preventing a retraction pocket and cholesteatoma recurrence in patients with a poorly functioning eustachian tube or a defective scutum, while preserving the same advantage of the canal wall up technique. Key Words: Bone pate-Cholesteatoma-Mastoidectomy-Obliteration.

Otol Neurotol 26:1107-1111, 2005.
The principal objective in cholesteatoma surgery is the complete eradication of the disease to produce a safe ear. Over the past years, this goal has been consistently achieved using the canal wall down technique (CWDT). However, this technique has several problems, such as difficulty with fitting a hearing aid and a higher rate of infection $(1,2)$. Although these problems can be avoided using the canal wall up technique (CWUT), the incidence of cholesteatoma recurrence is higher with CWUT than with CWDT $(3,4)$. In particular, when patients have a destructive scutum or poor eustachian tube function,

Address correspondence and reprint requests to Won-Sang Lee, M.D., Ph.D., Department of Otorhinolaryngology, Yonsei University College of Medicine, 134 Shinchon-dong, Seodaemun-gu Seoul, 120752, South Korea; E-mail: wsleemd@yumc.yonsei.ac.kr surgeons usually choose to perform the CWDT to prevent the occurrence of retraction pockets, despite the disadvantages of this technique.

The main cause of retraction pocket formation in the CWUT is the negative pressure, which is produced from the preserved air-filled mastoid cavity (5). A retraction pocket can develop when a poorly functioning eustachian tube or scutum defect exists, which results in the recurrence of cholesteatoma. To avoid this process, manipulations to make the eustachian tube orifice patent (6) or reconstruct the scutum with cartilage (7), bone (8), or alloplastic material (9) have been performed. However, these procedures have not been entirely successful. Surgical methods to minimize the dead space or to seal off the epitympanic space have also been reported (1014). However, none of these methods could satisfactorily prevent the recurrence of the retraction pocket. 
To prevent the formation of retraction pocket after CWUT in patients with poor eustachian tube function or a defective scutum, a new technique was designed in which the source of negative pressure and the space for the retraction pocket were completely removed. The connection between the mastoid cavity and the middle ear was blocked by obliterating the epitympanum and antrum with bone pate and obliterating the remaining mastoid cavity with abdominal fat. This report describes the long-term anatomic and functional results of the patients who underwent this procedure.

\section{PATIENTS AND METHODS}

\section{Selection of Cases and Follow-up}

The study group included 151 patients with cholesteatoma who underwent mastoid cavity and epitympanic obliteration technique at the Yonsei University Severance Hospital between April 1997 and July 2001. The total number of cholesteatoma cases during the period was 292, and 151 of these cases were selected. The remaining cases were operated on with conventional CWUT or CWDT technique. Of the selected cases, 21 patients had a history of a previous mastoidectomy. The average age of the patients was 42 years (range, 11-69 yr). This technique was applied to cases with a high chance of recurrent retraction pocket and cholesteatoma formation, including those with an adhesive drum indicating poor eustachian tube function $(\mathrm{n}=$ $52)$ and patients with a destructive scutum $(n=68)$. Thirty-one patients had both adhesive drum and destructive scutum. The type and extension of cholesteatoma are summarized in Table 1. The average postoperative follow-up period was 31 months, ranging from 18 to 49 months. The anatomic results were assessed on the basis of the state of the ear canal, the condition of the neotympanum, and the presence or absence of a postoperative retraction pocket or cholesteatoma. To this end, the external ear and drum were examined microscopically at regular intervals. A staged operation for hearing gain was performed on patients with healthy drums and well-aerated middle ear cavities between 11 and 18 months after the initial operation in a total of 56 patients. In 27 of 56 patients, the stapes was intact and Type II tympanoplasty using partial ossicular replacement prosthesis was performed. For the remaining 29 cases where the superstructure of the stapes was partially missing or only the footplate was remaining, Type III tympanoplasty using total ossicular replacement prosthesis was performed. The functional results were evaluated on the basis of the air-bone gap at three different

TABLE 1. Types and extension of cholesteatoma in patients who underwent the mastoid and epitympanic obliteration $(n=151)$

\begin{tabular}{lccccr}
\hline & \multicolumn{3}{c}{ Extension of cholesteatoma } & \\
\cline { 2 - 5 } $\begin{array}{l}\text { Type of } \\
\text { cholesteatoma }\end{array}$ & $\begin{array}{c}\text { Tympanic } \\
\text { cavity }\end{array}$ & Attic & Antrum & Mastoid & Total \\
\hline Attic & 0 & 17 & 23 & 28 & 68 \\
Tensa & 23 & 28 & 11 & 21 & 83 \\
Total & 23 & 45 & 34 & 49 & 151 \\
\hline
\end{tabular}

frequencies $(500,1,000$, and 2,000 Hz) obtained at 12 months after surgery.

\section{Surgical Techniques}

Under general anesthesia, a conventional postauricular incision was made. A rectangular periosteal flap was elevated. A separate suction line and a glass bottle were used for the collection of the bone pate. Using a suction irrigator and a cutting burr, healthy cortical bone pate was collected in the bottle. It was then mixed with povidone-iodine solution and dried with a swab. A complete mastoidectomy was performed with preservation of the posterior canal wall. The cholesteatoma sac, pathologic mucosa, incus, malleus head, and tensor fold were removed. The antrum and epitympanic space posterior to the cochleariform process and superior to the tympanic segment of the facial nerve was obliterated with bone pate. As a result, the mastoid cavity was sealed off from the middle ear. The defective scutum was also reconstructed with bone pate. Gelfoam (Upjohn Co., Kalamazoo, MI, U.S.A.) was used to support the bone pate and prevent it from falling into the eustachian tube (Fig. $1 A$ and $B$ ). We expected the anterior epitympanum in front of the cochleariform process to remain as a free space to be ventilated through the eustachian tube and become an air-filled space. However, if the function of the eustachian tube is inadequate or nonfunctional, this space may be contracted; consequently, retraction and accumulation of keratin debris may occur. To prevent such complications, the scutum was partially removed (Fig. $1 C$ ). Then, the anterior epitympanum was accessible through the external ear canal for dressing. Temporalis muscle fascia was placed on the bone pate to prevent bare bone exposure. The remaining mastoid cavity was obliterated with abdominal fat (Fig. 1D). Reconstruction of the eardrum was performed in the usual manner.

\section{RESULTS}

\section{Anatomic Result}

In 114 of 151 patients $(75.4 \%)$, the middle ear was well healed and aerated. Tympanic membrane perforation and drum adhesion developed in $10(6.6 \%)$ and 34 cases $(22.5 \%)$, respectively. No retraction pocket or cholesteatoma recurrence developed in any of the cases (Table 2). The average period required for the complete healing of drum after surgery was 15.3 days (range, 12-33 d). A photograph of the drum and a computed tomographic scan taken 18 months after surgery showed that the reconstructed scutum and bone pate were well maintained without absorption (Fig. 2).

\section{Surgical Complications}

Postauricular skin depression was the most common complication of this technique $(\mathrm{n}=31$ [20.5\%]). In 18 patients $(11.9 \%)$, the postauricular wound was infected, but healing was achieved with a careful dressing. In three patients (2.0\%), the bone pate used for obliteration was infected, which required the performance of CWDT and removal of the infected bone pate under general anesthesia. The three cases with a fistula between the obliterated mastoid cavity and postauricular skin were corrected with local repair. One patient with an external 
FIG. 1. Intraoperative view of the epitympanic and antrum obliteration with bone pate $(A)$ and its schematic diagram $(B-D)$. Antrum and epitympanic space was obliterated and the defective scutum was reconstructed with bone pate $(B)$. If the function of the eustachian tube is inadequate, the scutum is partially removed to prevent recurrence of retraction pocket $(C)$. The remaining mastoid cavity was further obliterated with abdominal fat $(D)$. SS, sigmoid sinus; MCF, middle cranial fossa dural plate; arrow, external ear canal.
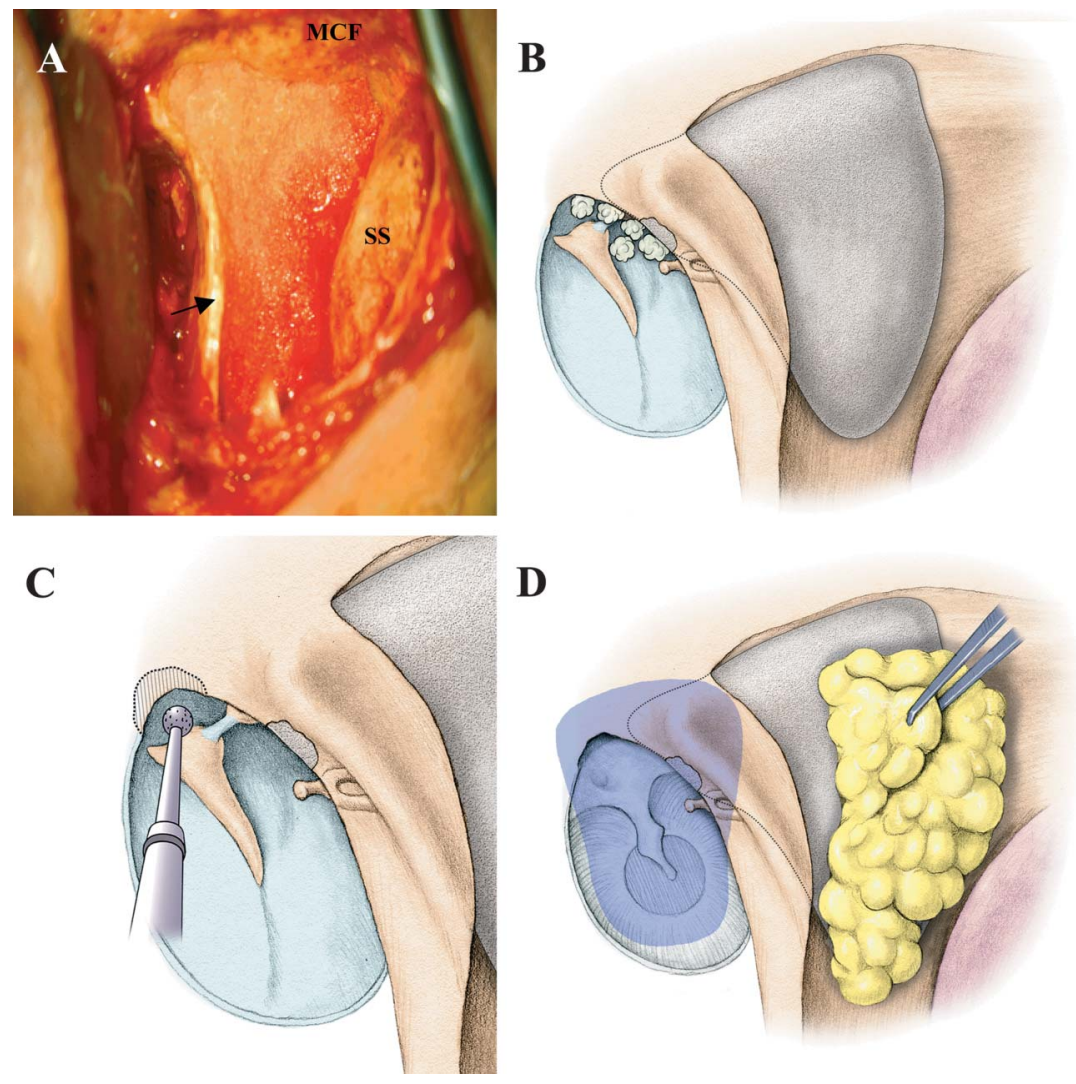

ear canal stenosis was treated with a local excision and skin graft. The complications during the follow-up period are listed in Table 3.

\section{Results of the Staged Operation}

Of the 56 cases who underwent a staged operation for hearing gain, 21 patients had postoperative air-bone gap less than $10 \mathrm{~dB}$ hearing level (HL). In 16 cases, the hearing fell into the group showing an air-bone gap between 10 and $20 \mathrm{~dB}$ HL. The postoperative air-bone gap was greater than $20 \mathrm{~dB}$ HL in the remaining 19 cases. Table 4 shows the air-bone gap and air conduction of the patients after staged operation. Residual cholesteatoma was discovered during the second procedure in 3 of 56 cases. In all three cases, the residual cholesteatoma was found in the form of a tiny skin

TABLE 2. Anatomic results after the mastoid and epitympanic obliteration technique $(n=151)$

\begin{tabular}{lc}
\hline \multicolumn{1}{c}{ Drum status } & No. of cases $(\%)$ \\
\hline Well-healed drum & $114(75.4)$ \\
Perforation & $10(6.6)$ \\
Adhesion & $34(22.5)$ \\
Retraction pocket & 0 \\
\hline
\end{tabular}

pearl in the middle ear cavity. In one patient, a bony particle was fixed to the ossicle.

\section{DISCUSSION}

The CWUT technique has many benefits compared with the CWDT, because the external auditory canal wall can be conserved. However, preservation of the pneumatized epitympanum and mastoid cavity creates conditions conducive to development of tympanic retraction pockets and recurrence of cholesteatoma. As pointed out by Palva and Virtanen (5), the more air-filled spaces there are, the higher the probability of retraction pockets. Accordingly, there have been several efforts aimed at reducing the air-filled mastoid cavity. Several surgeons have obliterated the mastoid cavity with abdominal fat or soft tissue after a CWUT and have reported slightly better results with respect to hearing and drum retraction compared with the air space reservoir technique (10-14). However, a retraction pocket still developed in the remaining epitympanic space in these techniques. Other surgeons have attempted to seal off the mastoid cavity with a bony septum at the antrum level, but the functional result was disappointing because of the absorption of bony septum, which resulted in an incomplete block between the middle ear and the mastoid cavity (11). 

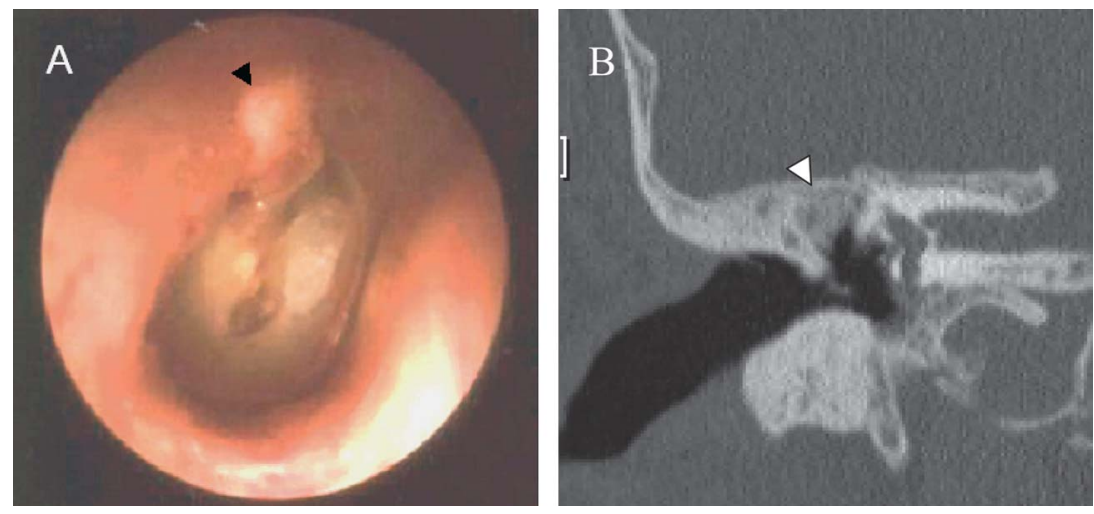

FIG. 2. Drum photograph $(A)$ and computed tomographic scan $(B)$ taken 18 months after surgery. The bone pate (arrowheads) used for the scutum reconstruction and epitympanic obliteration is well maintained without absorption.

The epitympanum and antrum were first obliterated with the bone pate and externalized for the purpose of both removing the space of the possible retraction pocket and sealing off the mastoid cavity from the middle ear. Subsequently, the mastoid cavity was further obliterated to remove the source of negative pressure. The formation of retraction pocket and the recurrence of cholesteatoma could be prevented using this procedure. The other anatomic results of this technique such as the period for postoperative healing, the incidence of residual cholesteatoma in the middle ear cavity, and the incidence of wound infections were not significantly different from those of the classic CWUT (15).

Hearing improvement is one of the main aims of middle ear surgery. Staged surgery for a second look and hearing gain was performed in 56 patients. Although the air reservoir space was eliminated, an adequate middle ear cavity for ossiculoplasty was retained. Therefore, the hearing results after staged surgery were satisfactory, considering that the preservation of preoperative hearing is indeed the most likely outcome after surgery for cholesteatoma $(16,17)$. However, this technique could not avoid the occurrence of an adhesive drum in cases where a eustachian tube failed to ventilate the middle ear cavity. The drum was adhesive to the middle ear mucosa in $22.5 \%$ of our patients who had poor tubal function. Nevertheless, no retraction pocket or cholesteatoma developed, and a healthy external ear canal could be achieved in these cases. Therefore, the more adequate conditions for fitting a hearing aid were provided compared with CWDT.

TABLE 3. Surgical complications after the mastoid and epitympanic obliteration technique $(n=151)$

\begin{tabular}{lc}
\hline \multicolumn{1}{c}{ Complications } & No. of cases $(\%)$ \\
\hline Retroauricular skin depression & $31(20.5)$ \\
Retroauricular wound infection & $18(11.9)$ \\
Abdominal wound infection & $4(2.6)$ \\
Bone pate infection & $3(2.0)$ \\
Fistula (mastoid cavity-skin) & $3(2.0)$ \\
Canal stenosis & $1(0.7)$ \\
\hline
\end{tabular}

There is some controversy among otologists regarding management of the mastoid cavity after CWUT. Holmquist (18) suggested that an air reservoir in the form of an open mastoidectomy cavity connecting with the middle ear is essential for preventing abrupt air pressure drops in the small middle ear space. In contrast, Palva and Virtanen (5) reported that the normal aeration of the middle ear was observed when the mastoid cavity was obliterated, and they concluded that such an air reservoir serves no purpose. In this study, complete removal of the air reservoir space was performed, and as a result, some of our patients complained of otalgia during flights and upper airway infections. These symptoms can be attributed to the decrease in their reservoir space, but there was no complications such as a retraction pocket or a drum perforation during flights and upper airway infection.

Autogenous bone pate was used to obliterate the attic space. The computed tomographic scans shows that the bone pate maintained its shape with time. We encountered three cases with bone pate infection in whom the removal of the bone pate was necessary. Therefore, careful infection control is mandatory before and after obliteration. There was a case with a canal stenosis and another case with an ossicle fixation caused by the bone pate. Surgeons need to take every precaution to prevent bone dust from entering the middle ear and external ear canal. Abdominal fat was used to obliterate the mastoid cavity, and $20.5 \%$ of the patients had a retroauricular skin depression. The reason might be that the volume of fat may have reduced with time.

TABLE 4. Hearing results after ossiculoplasty in patients who underwent mastoid and epitympanic obliteration $(n=56)$

\begin{tabular}{lcc}
\hline $\begin{array}{c}\text { Postoperative air-bone } \\
\text { gap (dB HL) }\end{array}$ & $\begin{array}{c}\text { Mean postoperative } \\
\text { air conduction of each } \\
\text { group (dB HL) }\end{array}$ \\
\hline Group A (0-10) & $21(37.4)$ & $25.3 \pm 9.2$ \\
Group B (10-20) & $16(34.1)$ & $39.2 \pm 11.2$ \\
Group C ( $>20)$ & $19(28.5)$ & $51.3 \pm 10.3$ \\
\hline
\end{tabular}




\section{CONCLUSION}

In conclusion, mastoid and epitympanic obliteration was performed in patients with a poor functioning eustachian tube or a defective scutum. According to the long-term results, the recurrence of a retraction pocket and cholesteatoma after a mastoidectomy could be prevented effectively while preserving the same advantage of the CWUT. Overall, this technique is a useful substitute for CWDT in patients with a defective scutum or a poor functioning eustachian tube if a surgeon has strong faith in complete saucerization of the disease.

\section{REFERENCES}

1. Smyth GD. Canal wall for cholesteatoma: up or down? Long-term results. Am J Otol 1985;6:1-2.

2. Palmgren O. Long-term results of open cavity and tympanomastoid surgery of the chronic ear. Acta Otolaryngol 1979;88:343-9.

3. Charachon R, Gratacap B, Tixier C. Closed versus obliteration technique in cholesteatoma surgery. Am J Otol 1988;9:286-92.

4. Brown JS. A ten-year statistical follow-up of 1142 consecutive cases of cholesteatoma: the closed vs. the open technique. Laryngoscope 1982;92:390-6.

5. Palva T, Virtanen H. Ear surgery and mastoid air cell system. Arch Otolaryngol 1981;107:71-3.

6. Wullstein H. Eustachian tube in tympanoplasty. Arch Otolaryngol 1960;71:408-10.
7. Weber PC, Gantz BJ. Cartilage reconstruction of the scutum defects in canal wall up mastoidectomies. Am J Otolaryngol 1998; 19:178-82.

8. Pfleiderer AG, Ghosh S, Kairinos N, Chaudhri F. A study of recurrence of retraction pockets after various methods of primary reconstruction of attic and mesotympanic defects in combined approach tympanoplasty. Clin Otolaryngol 2003;28: 548-51.

9. Geyer G, Dazert S, Helms J. Performance of ionomeric cement (Ionocem) in the reconstruction of the posterior meatal wall after curative middle-ear surgery. J Laryngol Otol 1997;111:1130-6.

10. Vartiainen E, Harma R. Mastoid obliteration in intact canal wall mastoidectomy. Clin Otolaryngol 1987;12:327-9.

11. Villarejo PL, Banos EC, Ramos J. The antrum exclusion technique in cholesteatoma surgery. J Laryngol Otol 1992;106:120-3.

12. Montandon P, Benchaou M, Guyot JP. Modified canal wall-up mastoidectomy with mastoid obliteration for severe chronic otitis media. ORL J Otorhinolaryngol Relat Spec 1995;57:198-201.

13. Honda Y. My tympanoplasty Otorhinolaryngology Tokyo 1992; 35(Suppl 5):1-127.

14. Yanagihara N, Gyo K, Sasaki Y, Hinohira Y. Prevention of recurrence of cholesteatoma in intact canal wall tympanoplasty. Am J Otol 1993;14:590-4.

15. Sheehy JL. The intact canal wall technique in the management of aural cholesteatoma. J Laryngol Otol 1970;84:1-31.

16. Tos M, Torben L. Hearing after surgery for cholesteatoma using various technique. Auris Nasus Larynx 1989;16:61-73.

17. Ragheb SM, Gantz BJ, Gabe BF. Hearing results after cholesteatoma surgery: the Iowa experience. Laryngoscope 1987;97: $1254-62$.

18. Holmoquist J, Bergstrom B. The mastoid air cell system in ear surgery. Arch Otolaryngol 1978;104:127-9. 